\title{
Tendência da Mortalidade por Doenças Cerebrovasculares no Brasil (1996-2015) e Associação com Desenvolvimento Humano e Vulnerabilidade Social
}

\author{
Cerebrovascular Disease Mortality Trend in Brazil (1996 To 2015) and Association with Human Development \\ Index and Social Vulnerability
}

Carlos Dornels Freire de Souza, ${ }^{1}$ Denilson José de Oliveira, ${ }^{2}$ Leonardo Feitosa da Silva, ${ }^{1}$ Camila Damasceno dos Santos, ${ }^{2}$ Monaliza Coelho Pereira, ${ }^{2}$ João Paulo Silva de Paiva, ${ }^{1}$ Thiago Cavalcanti Leal, ${ }^{1}$ Renato de Souza Mariano, ${ }^{3}$ Amanda Karine Barros Ferreira de Araújo, ${ }^{1}$ Jussara Almeida de Oliveira Baggio ${ }^{1}$

Universidade Federal de Alagoas - Campus Arapiraca, ${ }^{1}$ Arapiraca, AL - Brasil

Faculdade São Francisco de Juazeiro, ${ }^{2}$ Juazeiro, BA - Brasil

Instituto de Medicina Integral Professor Fernando Figueira - UPAE, ${ }^{3}$ Petrolina, RE - Brasil

\section{Resumo}

Fundamento: As doenças cerebrovasculares (DCBV) constituem a segunda causa de mortes no mundo.

Objetivo: Analisar a tendência da mortalidade por DCVB no Brasil (1996-2015) e associação com o índice de desenvolvimento humano (IDH) e o índice de vulnerabilidade social (IVS).

Métodos: Trata-se de estudo ecológico envolvendo as taxas de mortalidade padronizadas por DCBV. Os dados dos óbitos foram obtidos do Sistema de Informações sobre Mortalidade e os dados populacionais, do Instituto Brasileiro de Geografia e Estatística. Para as análises temporais, foi utilizado o modelo de regressão por pontos de inflexão, sendo calculado o percentual de variação anual (annual percent change [APC]) e médio do período (average annual percent change [AAPC]), com intervalo de confiança de $95 \%$ e significância de $5 \%$. As tendências foram classificadas em crescente, decrescente ou estacionária. $\mathbf{O}$ modelo de regressão multivariada foi utilizado para testar a associação entre a mortalidade por DCBV, IDH e IVS.

Resultados: Foram registrados 1.850 .811 óbitos por DCBV no período estudado. Observou-se redução da taxa de mortalidade nacional (APC: $-2,4 ; p=0,001$ ). Vinte unidades federativas apresentaram tendências significativas, sendo 13 de redução, incluindo todos das regiões Centro-Oeste $(n=4)$, Sudeste $(n=4)$ e Sul $(n=3)$. O IDH teve associação positiva e o IVS, associação negativa com a mortalidade ( $p=0,046$ e $p=0,026$, respectivamente).

Conclusão: $\mathrm{O}$ estudo mostrou comportamento epidemiológico desigual da mortalidade entre as regióes, sendo maior nos estados do Sudeste e Sul, porém com tendência significativa de redução, e menor nos estados do Norte e Nordeste, mas com tendência significativa de crescimento. O IDH e o IVS associaram-se com a mortalidade. (Arq Bras Cardiol. 2021; 116(1):89-99)

Palavras-chave: Encefalopatias/mortalidade; Epidemiologia; Desenvolvimento da Comunidade; Vulnerabilidade Social; Estudos de Séries Temporais; Morbimortalidade; Acidente Vascular Cerebral/mortalidade; Serviços Médicos de Emergência/organização e administração.

\footnotetext{
Abstract

Background: Cerebrovascular diseases (CBVD) are the second major cause of death in the world.

Objective: To analyze the mortality trend of CBVD in Brazil (1996 to 2015) and its association with Human Development Index (HDI) and the Social Vulnerability Index (SVI).

Methods: This is an ecological study. We analyzed the mortality rate standardized by CBVD. Death data were obtained from the Mortality Information System (SIM) and populational data from the Brazilian Institute of Geography and Statistics (IBGE). The model of regression by inflection points (Joinpoint regression) was used to perform the temporal analysis, calculating the Annual Percent Change (APC) and Average Annual Percent Change (AAPC), with 95\% of confidence interval and a significance of 5\%. Trends were classified as increasing, decreasing or stationary. A multivariate regression model was used to analyze the association between mortality by CBVD, HDI and SVI.
}

Correspondência: Carlos Dornels Freire de Souza •

Universidade Federal de Alagoas - Campus Arapiraca - Medicina - Av. Manoel Severino Barbosa. CEP 57309-005, Arapiraca, AL - Brasil

E-mail: carlos.freire@arapiraca.ufal.br

Artigo recebido em 08/08/2019, revisado em 05/11/2020, aceito em 27/12/2019

DOI: https://doi.org/10.36660/abc.20190532 
Results: During this period, 1,850,811 deaths by CBVD were recorded. We observed a reduction in the national mortality rate (APC -2.4 ; $p=$ 0.001). Twenty federation units showed a significant trend, of which 13 showed reduction, including all states in the Midwest ( $n=4$ ), Southeast $(n=4)$ and South $(n=3)$. The HDI was positively associated and the SVI was negatively associated with mortality $(p=0.046$ and $p=0.026$, respectively).

Conclusion: An unequal epidemiological course of mortality was observed between the regions, being higher in the Southeast and South states, with a significative tendency of reduction, and lower in the North and Northeast states, but with a significative tendency of increase. HDI and SVI showed an association with mortality. (Arq Bras Cardiol. 2021; 116(1):89-99)

Keywords: Brain Diseases/mortality; Epidemiology; Community Development; Social Vulnerability; Time Seies Studies; Morbimortality; Stroke/ mortality; Emergency Medical Emergencies/organization and administration.

Full texts in English - http://www.arquivosonline.com.br

\section{Introdução}

As doenças crônicas não transmissíveis (DCNT) têm ocupado lugar de destaque no cenário epidemiológico, representando atualmente o maior problema global de saúde, causando cerca de 38 milhões de mortes anualmente (70\% de todos os óbitos), sendo 16 milhões delas consideradas prematuras (idade inferior a 70 anos). ${ }^{1}$ No Brasil, aproximadamente $75 \%$ dos óbitos são causados pelas DCNT, o que representa mais de 1 milhão de mortes a cada ano. ${ }^{2}$

O grupo das DCNT é composto por quatro subgrupos: cardiovasculares (DCV), câncer, doença respiratória crônica e diabetes melito. Dentre as DCV, destacam-se as doenças cerebrovasculares (DCBV), que figuram como a segunda causa de mortalidade no mundo, ficando atrás apenas das doenças isquêmicas do coração. Juntas, elas foram responsáveis por 15,2 milhões de mortes em 2016. ${ }^{1,3}$

Dentre os países da América Latina, o Brasil apresenta uma das maiores taxas de mortalidade por DCBV. Nas últimas décadas, houve um aumento expressivo do número de óbitos, passando de 104 mil, em 1990, para 144 mil, em 2015. Por outro lado, o país tem experimentado uma redução da taxa de mortalidade, sobretudo a precoce, que decresceu de 51,4\% em 1990, para 35,1\% em 2015. ${ }^{4}$

Considerando que o impacto das DCBV na morbimortalidade é um desafio para o desenvolvimento econômico e social das nações, sobretudo nos países em desenvolvimento, os quais concentram cerca de $80 \%$ de todos os óbitos, ${ }^{1,5}$ o monitoramento do comportamento temporal dos indicadores no Brasil, um país de dimensões continentais e com importantes desigualdades socioespaciais, é de fundamental importância para a definição de políticas públicas que possam impactar na situação de saúde da população. ${ }^{6}$

Nesse sentido, este trabalho objetivou analisar a tendência da mortalidade por DCVB no Brasil (1996-2015) e a associação com o índice de desenvolvimento humano (IDH) e o índice de vulnerabilidade social (IVS).

\section{Métodos}

\section{Desenho do estudo, população e período}

Trata-se de um estudo ecológico envolvendo todos os óbitos DCBV ocorridos no Brasil no período de 1996 a 2015 e o IDH e o IVS. Adotou-se como unidades de análise o Brasil, suas grandes regiões e as unidades da federação.

\section{Variáveis estudadas}

Foram analisadas variáveis sociodemográficas: gêmero (masculino, feminino e ignorado), faixa etária em anos (0-4, 5-9, 10-14, 15-19, 20-29, 30-39, 40-49, 50-59, 60-69, 70-79, 80 e mais e idade ignorada), escolaridade em anos (nenhuma, 1-3, 4-7, 8-11, 12 ou mais e escolaridade ignorada) e estado civil (solteiro, casado, viúvo, separado, outro e estado civil ignorado). Para a análise de série temporal, foi incluída a variável taxa de mortalidade padronizada por idade e sexo em decorrência das DCBV. Para o componente de associação, foram selecionados dois índices sociais: i) o IDH e suas três dimensões (longevidade, educação e renda) e ii) o IVS e suas três dimensões (infraestrutura urbana, capital humano e renda e trabalho). Esses dois índices mensuram, respectivamente, o grau de desenvolvimento humano e o grau de vulnerabilidade social a que uma população está exposta.

\section{Fonte de dados e coleta}

Os dados dos óbitos foram coletados do Sistema de Informações sobre Mortalidade (SIM) do Ministério da Saúde (http://datasus.saude.gov.br/). ${ }^{7}$ Considerou-se o Código Internacional de Doenças (CID-10) I60 a I69 - I60: hemorragia subaracnoide; 161: hemorragia intracerebral; 162: outras hemorragias intracranianas não traumáticas; 163: infarto cerebral; 164: acidente vascular cerebral (AVC) não especificado como hemorrágico ou isquêmico; 165: oclusão/ estenose de artérias pré-cerebrais que não resultam em infarto cerebral; 166: oclusão/estenose de artérias cerebrais que não resultam em infarto cerebral; 167: outras doenças cerebrovasculares; 168: transtornos cerebrovasculares em doenças classificadas em outra parte; 169: sequelas de doenças cerebrovasculares. ${ }^{8}$ Os dados populacionais necessários ao cálculo dos indicadores foram obtidos do Instituto Brasileiro de Geografia e Estatística (IBGE). ${ }^{9}$

Para a obtenção das taxas, foram utilizadas as seguintes equações:

a) Taxa de mortalidade anual: no de óbitos por DCBV no local e ano/população no local e ano × 100.000 habitantes.

b) Taxa de mortalidade do período (1996-2015): média de óbitos por DCBV da série temporal (1996-2015)/população do meio do período $\times 100.000$.

Por fim, o IDH foi obtido do atlas de desenvolvimento humano (http://atlasbrasil.org.br/2013/) e o IVS, do atlas de vulnerabilidade social (http://ivs.ipea.gov.br/index.php/pt/), tendo como base o ano de 2010. Salienta-se que os dados de IDH e IVS somente são calculados nos anos censitários. 


\section{Padronização das taxas de mortalidade}

Para a redução dos efeitos da estrutura demográfica populacional, as taxas brutas foram padronizadas por gênero e idade pelo método direto, considerando-se como população padrão a brasileira do ano de 2010 (ano censitário) e as seguintes faixas etárias: 0-4, 5-9, 10-14, 15-19, 20-29, 30-39, $40-49,50-59,60-69,70-79$ e 80 ou mais.

\section{Tratamento estatístico}

Para a análise temporal, foi utilizado o modelo de regressão por pontos de inflexão (joinpoint regression model). O modelo testa se uma linha com múltiplos segmentos é mais adequada para explicar o comportamento temporal de um conjunto de dados quando comparada com uma linha reta ou com menos segmentos. Desse modo, o joinpoint possibilita identificar a tendência de cada indicador (se estacionária, crescente ou decrescente), os pontos no tempo em que há modificação nessa tendência (joins), bem como a variação percentual anual (annual percent change [APC]) e do período total (average annual percent change [AAPC]). ${ }^{10} \mathrm{Na}$ configuração do modelo, foram adotados os seguintes parâmetros: número mínimo de joins: zero; número máximo de joins: três; seleção do melhor modelo: teste de permutação de Monte Carlo ( $n=4.499$ permutações); método de autocorrelação dos erros: método baseado na data; intervalo de confiança: 95\% (IC 95\%); e nível de significância: 5\%.

Para a análise de associação entre os indicadores sociais e a taxa padronizada de mortalidade, adotou-se o modelo de regressão multivariada (ordinary least square [OLS]).

Para as análises, foram utilizados os softwares Joinpoint Regression 4.5.0.1 (National Cancer Institute, USA), GeoDa 1.10.0.8 (Universityof Illinois at Urbana- Champaign, USA) e QGis 2.14.11 (Open Source Geospatial Foundation, USA). As malhas territoriais necessárias para a confecção dos mapas foram provenientes do IBGE.

\section{Aspectos éticos}

Por utilizar dados secundários de domínio público, nos quais não é possível a identificação dos sujeitos, dispensou-se a apreciação do Comitê de Ética em Pesquisa.

\section{Resultados}

Entre 1996 e 2015, foram registrados 1.850.811 óbitos por DCBV no Brasil, expressando uma média de 92.540 casos/ano. Desse total, 50,68\% ( $n=938.044)$ ocorreram em indivíduos do gênero masculino e $77,80 \%(n=1.440 .170)$ em idosos. Ao estratificar segundo gênero, observou-se que a única faixa etária na qual a proporção de mulheres ultrapassou a de homens foi em indivíduos com idade igual ou superior a 80 anos. Destacou-se ainda a baixa escolaridade, uma vez que 39,94\% ( $n=739.233$ ) eram analfabetos ou tinham até três anos de estudo. Nessa variável, observou-se uma elevada proporção de campos ignorados $(38,29 \% / n=708.685)$ (Tabela 1 ).

Ao longo da série temporal, a taxa de mortalidade por DCBV na população brasileira, considerando ambos os gêneros, apresentou tendência linear de redução (APC $-2,4 \%$; IC 95\% -2,7 a -2,0; $p=0,001$ ), passando de 72,3/100 mil (1996) para 46,4/100 mil (2015). Comportamentos semelhantes foram observados na população masculina (APC - 2,3\%; IC 95\% -2,6 a -1,9; $\mathrm{p}=0,001$ ) e na feminina (APC -2,4\%; IC 95\% -2,8 a -2,0; p = 0,001), cujas taxas reduziram de 77,8 e 71,4/100 mil para 51,1 e 45,2/100 mil, respectivamente (Figura 1 ).

Ainda conforme a Figura 1, observa-se que a distribuição espacial das taxas médias é heterogênea, sendo maior nos estados das regiões Sudeste e Sul e menor no Norte. As maiores taxas médias gerais foram observadas nos estados do Paraná (75/100 mil) e Espírito Santo (71,3/100 mil), e as menores nos estados do Rio Grande do Norte (40,9/100 mil) e Bahia (48,0/100 mil). Esse mesmo cenário se repetiu para a mortalidade masculina (Paraná com 83,4/100 mil e Espírito Santo com 79,8/100 mil). Na população feminina, as maiores taxas foram observadas no Paraná (71,2/100 mil) e Rio Grande do Sul (69,2/100 mil) e as menores no Rio Grande do Norte $(40,7 / 100$ mil) e Bahia $(49,1 / 100$ mil).

$\mathrm{Na}$ etapa seguinte, analisou-se a tendência das taxas de mortalidade considerando toda a série temporal completa (1996-2015). A região Norte foi a única que apresentou tendência de crescimento da mortalidade na população geral (APC 0,4\%; IC 95\% 0,1 a 0,8; p < 0,001) e masculina (APC $0,7 \%$; IC 95\% 0,3 a 1,1; $p<0,001)$. As regiões Centro-Oeste, Sudeste e Sul apresentaram tendência decrescente, tanto na população geral quanto na masculina e feminina, com destaque para a região Sudeste com maior redução percentual na (APC 3,8\%) (Tabela 2).

Na análise estratificada por unidade da federação, 20 estados apresentaram tendências significativas, sendo sete de crescimento e 13 de redução. Todos os estados das regiões Centro-Oeste, Sul e Sudeste apresentaram tendências decrescentes, com destaque para o Rio de Janeiro e Santa Catarina, com os maiores percentuais de redução. Por outro lado, dos sete estados com tendência de crescimento, cinco estão situados na região nordeste (Maranhão, Piauí, Paraíba, Alagoas e Sergipe) e dois no Norte (Amazonas e Tocantins) (Tabela 2).

Quanto ao IDH, apenas o Distrito Federal foi classificado como muito alto (IDH 0,824). Todos os estados do Nordeste e cinco do Norte apresentaram IDH médio (entre 0,600 e 0,699), destacando-se Alagoas e Maranhão com valores mais baixos (IDH 0,631 e 0,639, respectivamente). Em paralelo, esses mesmos estados das regiões Norte e Nordeste apresentaram os maiores valores no IVS, destacando-se o Maranhão com IVS muito alto (IVS 0,521). Todos os oito estados classificados como alta vulnerabilidade social estão situados nas regiões Norte (n $=4)$ e Nordeste $(n=4)$ (Figura 2).

O modelo de regressão temporal mostrou ainda que os estados das regiões Norte e Nordeste foram os que apresentaram o maior número de segmentos na série temporal (joins), representando maior oscilação nas taxas ao longo dos anos. Ao analisar a mortalidade geral no Nordeste, por exemplo, quatro segmentos temporais foram encontrados: crescimento discreto (1996-2003), comportamento estacionário (2003-2006), tendência de redução (2006-2010) e novamente um comportamento estacionário (2010-2015). Dentre os estados dessa região, apenas a Bahia apresentou comportamento linear (Tabela 3). 


\begin{tabular}{|c|c|c|c|c|c|c|c|c|}
\hline \multirow[t]{2}{*}{ Variáveis } & \multicolumn{2}{|c|}{$\begin{array}{c}\text { Masculino } \\
n=938.044 \\
(50,68 \%)\end{array}$} & \multicolumn{2}{|c|}{$\begin{array}{c}\text { Feminino } \\
n=912.202 \\
(49,29 \%)\end{array}$} & \multicolumn{2}{|c|}{$\begin{array}{l}\text { Ignorado } \\
n=565 \\
(0,03 \%)\end{array}$} & \multicolumn{2}{|c|}{$\begin{array}{l}\text { Total de Óbitos } \\
n=1.850 .811 \\
(100 \%)\end{array}$} \\
\hline & $\mathrm{n}$ & $\%$ & $\mathrm{n}$ & $\%$ & $\mathrm{n}$ & $\%$ & $\mathrm{n}$ & $\%$ \\
\hline \multicolumn{9}{|l|}{ Faixa etária } \\
\hline $0-4$ & 1.012 & 55,95 & 793 & 43,83 & 4 & 0,22 & 1.809 & 1,00 \\
\hline $5-9$ & 565 & 53,25 & 496 & 46,75 & 0 & 0,00 & 1.061 & 0,06 \\
\hline $10-14$ & 999 & 54,44 & 834 & 45,45 & 2 & 0,11 & 1.835 & 0,10 \\
\hline $15-19$ & 1.998 & 55,27 & 1.616 & 44,70 & 1 & 0,03 & 3.615 & 0,20 \\
\hline $20-29$ & 7.158 & 52,66 & 6.426 & 47,27 & 10 & 0,07 & 13.594 & 0,73 \\
\hline $30-39$ & 21.278 & 50,09 & 21.186 & 49,87 & 17 & 0,04 & 42.481 & 2,30 \\
\hline $40-49$ & 62.652 & 50,98 & 60.217 & 48,99 & 37 & 0,03 & 122.906 & 6,64 \\
\hline $50-59$ & 124.934 & 56,74 & 95.185 & 43,23 & 65 & 0,03 & 220.184 & 11,90 \\
\hline $60-69$ & 200.551 & 57,92 & 145.578 & 42,05 & 106 & 0,03 & 346.235 & 18,71 \\
\hline $70-79$ & 268.228 & 53,12 & 236.627 & 46,85 & 135 & 0,03 & 504.990 & 27,28 \\
\hline 80 e mais & 246.717 & 41,89 & 342.104 & 58,09 & 124 & 0,02 & 588.945 & 31,81 \\
\hline Idade ignorada & 1.952 & 61,85 & 1.140 & 36,12 & 64 & 2,03 & 3.156 & 0,17 \\
\hline \multicolumn{9}{|l|}{ Escolaridade } \\
\hline Nenhuma & 162.163 & 42,91 & 215.672 & 57,07 & 90 & 0,02 & 377.925 & 20,42 \\
\hline $1-3$ anos & 192.038 & 53,15 & 169.257 & 46,84 & 13 & 0,01 & 361.308 & 19,52 \\
\hline 4-7 anos & 126.285 & 53,86 & 108.156 & 46,13 & 11 & 0,01 & 234.452 & 12,67 \\
\hline 8-11 anos & 54.461 & 54,17 & 46.075 & 45,83 & 6 & 0,01 & 100.542 & 5,43 \\
\hline 12 anos e mais & 29.083 & 57,64 & 21.369 & 42,35 & 4 & 0,01 & 504.56 & 2,73 \\
\hline $1-8$ anos $^{*}$ & 4.868 & 53,92 & 4.158 & 46,06 & 2 & 0,02 & 9.028 & 0,49 \\
\hline 9-11 anos* & 4.551 & 54,08 & 3.860 & 45,87 & 4 & 0,05 & 8.415 & 0,45 \\
\hline Ignorado & 364.595 & 51,45 & 343.655 & 48,49 & 435 & 0,06 & 708.685 & 38,29 \\
\hline \multicolumn{9}{|l|}{ Estado Civil } \\
\hline Solteiro & 163.672 & 47,22 & 182.828 & 52,75 & 87 & 0,03 & 346.587 & 18,73 \\
\hline Casado & 499.651 & 67,31 & 242.565 & 32,67 & 143 & 0,02 & 742.359 & 40,11 \\
\hline Viúvo & 152.794 & 28,22 & 388.484 & 71,76 & 124 & 0,02 & 541.402 & 29,25 \\
\hline Separado & 40.958 & 59,29 & 28.116 & 40,70 & 6 & 0,01 & 69.080 & 3,73 \\
\hline Outro & 12.235 & 64,02 & 6.875 & 35,97 & 1 & 0,01 & 19.111 & 1,03 \\
\hline Ignorado & 68.734 & 51,96 & 63.334 & 47,88 & 204 & 0,16 & 132.272 & 7,15 \\
\hline
\end{tabular}

*Diferentes agrupamentos de escolaridade se devem às mudanças ocorridas no formulário de declaração de óbito (DO) em 2011.

Por fim, o modelo de regressão mostrou associação positiva entre a taxa média de mortalidade e o IDH municipal $(p=0,046)$ e sua dimensão renda $(p=0,029)$, e associação negativa com o IVS ( $p=0,026)$ e em duas dimensões: capital humano $(p=0,046)$ e renda e trabalho $(p=0,018)$ (Tabela 4$)$.

\section{Discussão}

O Brasil apresenta uma das maiores taxas de mortalidade por DCBV dentre os países da América Latina e ainda muito superior ao de nações desenvolvidas. ${ }^{11}$ Todavia, um comportamento temporal de declínio da taxa nacional tem sido observado ao longo das últimas décadas, ${ }^{1}$ tanto na população masculina quanto na feminina, corroborando a literatura existente nacional e internacional. ${ }^{12-15}$

Muitos autores têm destacado que tal redução da mortalidade pode ser explicada pela ampliação do acesso aos serviços de saúde e da adoção de estratégias de prevenção. ${ }^{14,15}$ No Brasil, destaca-se a implantação da atenção primária à saúde (APS), que, por meio da Estratégia Saúde da Família (ESF) desenvolve ações de controle de fatores de risco, a exemplo do incentivo à prática de atividade física e da adoção de hábitos alimentares salutares, programa de controle do tabagismo, diagnóstico e acompanhamento sistemático das condições crônicas (p. ex., hipertensão e diabetes) e acesso 
A - Taxa de Mortalidade por DCBV na população geral. Brasil, 1996-2015.

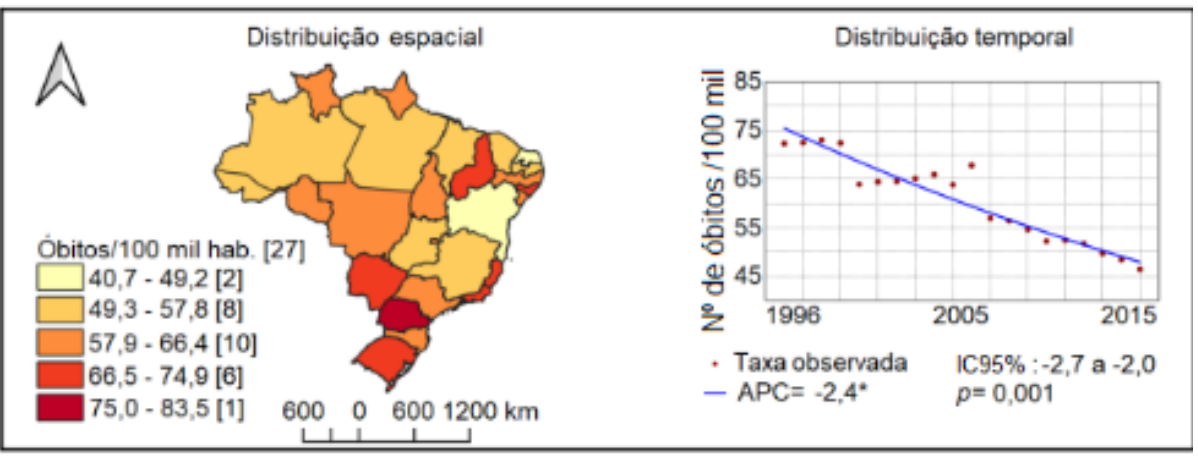

B - Taxa de Mortalidade por DCBV em Homens. Brasil, 1996-2015.

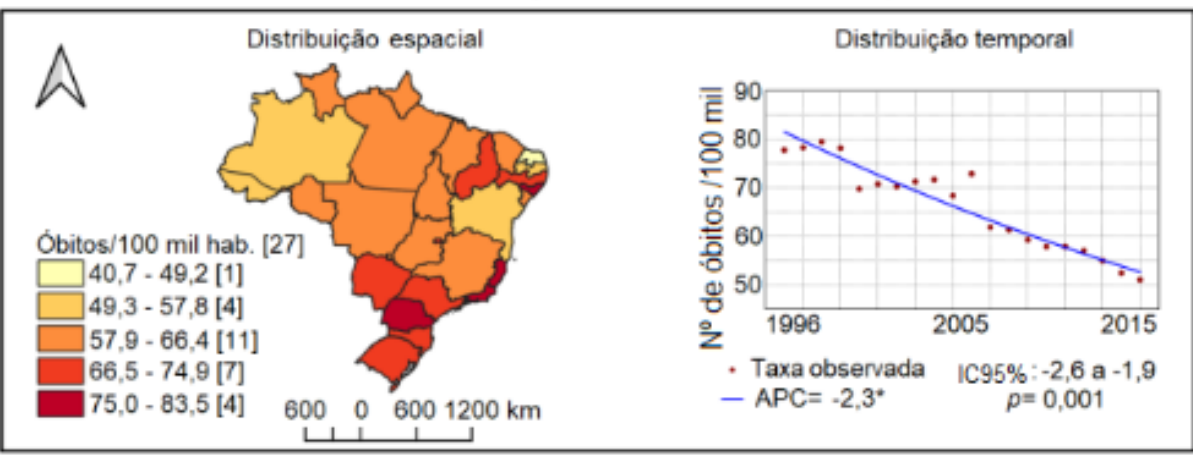

C - Taxa de Mortalidade por DCBV em Mulheres. Brasil, 1996-2015.

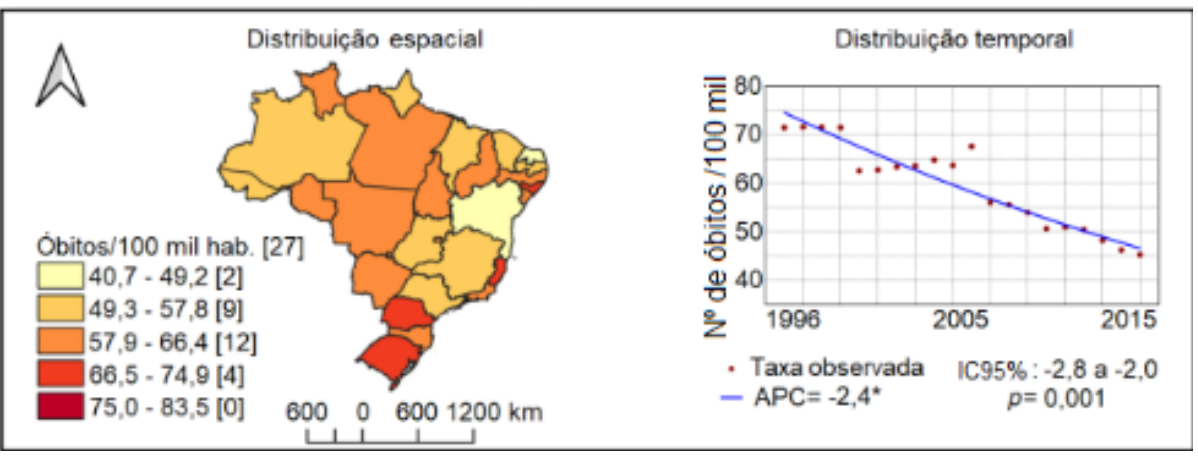

Figura 1 - Distribuição espacial e tendência das taxas de mortalidade padronizada por DCBV nos estados brasileiros, geral e segundo gênero. Brasil (1996-2015). APC: Annual Percent Change; hab.: habitantes; $n^{\circ}$ : número; IC95\%: intervalo de confiança de 95\%; DCBV: doenças cerebrovasculares.

à assistência farmacêutica. ${ }^{16,17}$ Entre 1998 e 2017, houve uma intensa expansão do número de equipe de saúde da família, passando de pouco mais de 2 mil para 41 mil, alcançando uma cobertura de $70 \%$ da população brasileira, o que corresponde a aproximadamente 143 milhões de pessoas. ${ }^{17,18}$ Estudos têm mostrado associação entre a expansão da atenção primária e a redução da mortalidade por doenças como infarto agudo do miocárdio e doenças cerebrovasculares. ${ }^{19}$

Além da APS, o Brasil também avançou consideravelmente no atendimento ao paciente com DCBV. Em 1997, foi implantada a primeira unidade de AVC no Brasil, situada em Joinville-SC. A partir dessa experiência, em 2008, o Ministério da Saúde iniciou a organização da rede nacional de atendimento ao AVC, resultando na promulgação da portaria no 665/2012, com a finalidade de implantar serviços de referência em todo o país. ${ }^{20,21}$

Outra ação importante diz respeito ao Plano de Ações Estratégicas para o Enfrentamento das DCNT. Implantado em 2011 pelo Ministério da Saúde, o plano estabeleceu um conjunto de metas para o país, como a redução da mortalidade prematura por DCNT, da prevalência de tabagismo e do consumo de álcool na população, aumento da prevalência da prática de atividade física e do consumo de frutas e contenção do crescimento da obesidade. ${ }^{22}$

$\mathrm{Na}$ análise regional, evidenciamos um comportamento heterogêneo no padrão de mortalidade pelas DCBV no país, 
Tabela 2 - Percentual de variação média anual (AAPC) das taxas de mortalidade padronizadas por DCBV, segundo gênero, no Brasil, regiões e unidades federadas (1996-2015)

\begin{tabular}{|c|c|c|c|c|c|c|c|c|c|}
\hline \multirow{3}{*}{$\begin{array}{l}\text { Unidade } \\
\text { espacial }\end{array}$} & \multicolumn{3}{|c|}{ Ambos os gêneros } & \multicolumn{3}{|c|}{ Masculino } & \multicolumn{3}{|r|}{ Feminino } \\
\hline & \multicolumn{2}{|c|}{$\operatorname{Taxa}^{1}$} & \multirow{2}{*}{ APC (IC 95\%) p valor } & \multicolumn{2}{|c|}{$\operatorname{Taxa}^{1}$} & \multirow{2}{*}{ AAPC (IC 95\%) p valor } & \multicolumn{2}{|c|}{$\operatorname{Taxa}^{1}$} & \multirow{2}{*}{ AAPC (IC 95\%) p valor } \\
\hline & 1996 & 2015 & & 1996 & 2015 & & 1996 & 2015 & \\
\hline Norte & 50,5 & 58,6 & $0,4^{*}(0,1$ a 0,8$) ; p<0,001$ & 51,0 & 61,6 & $0,7^{*}(0,3$ a 1,1$) ; p<0,001$ & 52,6 & 58,6 & $0,1(-0,3$ a 0,5$) ; p=0,6$ \\
\hline RO & 68,2 & 51,4 & $-1,8^{*}(-2,2 a-1,8) ; p<0,001$ & 65,9 & 53,3 & $1,6^{*}(-2,3 a-1,0) ; p<0,001$ & 74,2 & 51,5 & $-1,9^{*}(-2,3$ a $-1,4) ; p<0,001$ \\
\hline$A C$ & 57,4 & 63,1 & $-0,1(-1,9$ a 1,7$) ; p=0,9$ & 59,5 & 62,7 & $0,4(-2,3$ a 3,1$) ; p=0,8$ & 58,7 & 66,9 & $0,4(-4,3$ a 5,4$) ; p=0,9$ \\
\hline AM & 49,7 & 56,1 & $0,6^{*}(0,2$ a 1,1$) ; p<0,001$ & 49,7 & 58,0 & $0,9^{*}(0,2$ a 1,5$) ; p<0,001$ & 52,7 & 57,3 & $0,4(-0,1$ a 1,0$) ; p=0,1$ \\
\hline $\mathrm{RR}$ & 75,5 & 46,8 & $-2,2^{*}(-3,0$ a $-1,3) ; p<0,001$ & 92,0 & 50,7 & $-2,0^{*}(-3,6$ a $-0,4) ; p<0,001$ & 56,4 & 44,2 & $-2,3^{*}(-3,3 a-1,3) ; p<0,001$ \\
\hline PA & 46,3 & 61,8 & $1,2(-0,8$ a 3,4$) ; p=0,2$ & 45,7 & 66,0 & $1,9(-0,3$ a 4,1$) ; p=0,1$ & 49,5 & 60,8 & $0,1(-2,0$ a 2,2$) ; p=0,9$ \\
\hline AP & 79,5 & 49,2 & $-1,7(-7,8$ a 4,9$) ; p=0,6$ & 77,1 & 54,3 & $-0,8(-4,8$ a 3,5$) ; p=0,7$ & 86,2 & 46,6 & $-1,2(-3,8$ a 1,5$) ; p=0,4$ \\
\hline TO & 43,4 & 59,3 & $1,9^{*}(0,9$ a 2,9$) ; p<0,001$ & 48,0 & 60,1 & $1,4(-1,3$ a 4,3$) ; p=0,3$ & 40,0 & 60,7 & $2,3^{*}(0,7$ a 3,9$) ; p<0,001$ \\
\hline Nordeste & 45,4 & 54,4 & $0,9(-0,7$ a 2,4$) ; p=0,3$ & 46,8 & 60,7 & $1,3(-0,3$ a 2,9$) ; p=0,1$ & 46,7 & 52,7 & $0,6(-1,1$ a 2,3$) ; p=0,5$ \\
\hline MA & 29,0 & 68,2 & $4,6^{*}(2,0$ a 7,4$) ; p<0,001$ & 31,7 & 76,6 & $4,7^{*}(2,5$ a 7,0$) ; p<0,001$ & 27,2 & 64,8 & $4,3^{*}(1,6$ a 7,0$) ; p<0,001$ \\
\hline $\mathrm{PI}$ & 33,3 & 76,9 & $3,9^{*}(2,9$ a 4,8$) ; p<0,001$ & 35,0 & 90,4 & $4,2^{*}(2,9$ a 5,5$) ; p<0,001$ & 33,0 & 70,3 & $4,0^{*}(3,0$ a 4,9$) ; p<0,001$ \\
\hline CE & 42,0 & 55,1 & $1,3(-0,2$ a 2,8$) ; p=0,1$ & 43,5 & 62,6 & $1,7^{*}(0,2$ a 3,2$) ; p<0,001$ & 42,8 & 52,1 & $0,7(-0,2$ a 1,6$) ; p=0,1$ \\
\hline RN & 33,0 & 38,0 & $0,9(-0,1$ a 1,8$) ; p=0,1$ & 34,4 & 43,7 & $1,3^{*}(0,2$ a 2,5$) ; p<0,001$ & 33,0 & 35,6 & $-0,4(-0,8$ a 1,5$) ; p=0,5$ \\
\hline PB & 37,5 & 48,5 & $1,7^{\star}(0,4$ a 3,0$) ; p<0,001$ & 39,0 & 52,2 & $1,9^{*}(0,3$ a 3,4$) ; p<0,001$ & 38,4 & 48,6 & $1,4(-0,1$ a 2,8$) ; p=0,1$ \\
\hline PE & 64,8 & 58,0 & $-0,8(-2,2$ a 0,6$) ; p=0,3$ & 68,1 & 66,6 & $-0,4(-1,0$ a 0,2$) ; p=0,2$ & 65,9 & 55,2 & $-1,1(-2,6$ a 0,4$) ; p=0,1$ \\
\hline $\mathrm{AL}$ & 55,5 & 69,3 & $0,8^{*}(0,2$ a 1,5$) ; p<0,001$ & 57,8 & 77,7 & $1,2^{*}(0,6$ a 1,8$) ; p<0,001$ & 57,1 & 66,9 & $0,5(-0,5$ a 1,5$) ; p=0,3$ \\
\hline SE & 41,8 & 57,6 & $1,7^{\star}(1,0$ a 2,3$) ; p<0,001$ & 45,7 & 64,7 & $1,9^{*}(1,0$ a 2,9$) ; p<0,001$ & 40,2 & 55,6 & $1,5^{*}(0,5$ a 2,5$) ; p<0,001$ \\
\hline BA & 47,7 & 45,2 & $-0,0(-0,6$ a 0,5$) ; p=0,9$ & 47,4 & 47,5 & $0,2(-0,3$ a 0,8$) ; p=0,4$ & 51,0 & 46,2 & $-0,2(-0,8$ a 0,3$) ; p=0,4$ \\
\hline Centro-Oeste & 69,5 & 46,3 & $-2,8^{\star}(-3,4 a-2,2) ; p<0,001$ & 72,2 & 49,2 & $-2,7^{*}(-3,3$ a $-2,2) ; p<0,001$ & 69,1 & 46,2 & $-2,3(-4,8$ a 0,2$) ; p=0,1$ \\
\hline MS & 76,9 & 52,9 & $-2,4^{*}(-2,9 a-2,0) ; p<0,001$ & 83,9 & 54,5 & $-2,4^{*}(-3,0$ a $-1,8) ; p<0,001$ & 73,1 & 54,5 & $-2,4^{*}(-2,8$ a 1,9$) ; p<0,001$ \\
\hline MT & 65,7 & 44,2 & $-1,9^{*}(-3,0$ a $-0,8) ; p<0,001$ & 66,3 & 45,6 & $-2,1^{*}(-3,5$ a $-0,6) ; p<0,001$ & 67,6 & 44,8 & $-2,5^{*}(-3,2$ a $-1,9) ; p<0,001$ \\
\hline GO & 64,2 & 46,2 & $-2,2^{*}(-2,6$ a $-1,8) ; p<0,001$ & 66,0 & 49,7 & $-1,6^{*}(-2,7$ a $-0,4) ; p<0,001$ & 65,1 & 45,6 & $-2,2(-2,6$ a $-1,8) ; p<0,001$ \\
\hline DF & 81,3 & 41,6 & $-4,0^{*}(-4,6$ a $-3,5) ; p<0,001$ & 91,6 & 46,4 & $-4,0^{*}(-4,6$ a $-3,4) ; p<0,001$ & 77,2 & 40,5 & $-3,4(-5,4$ a $-1,3) ; p<0,001$ \\
\hline Sudeste & 86,1 & 41,4 & $-3,8^{*}(-4,1$ a $-3,4) ; p<0,001$ & 96,3 & 45,8 & $-3,8^{*}(-4,2$ a $-3,5) ; p<0,001$ & 82,3 & 40,2 & $-3,8^{*}(-4,2$ a $-3,4) ; p<0,001$ \\
\hline MG & 74,1 & 39,3 & $-3,2^{*}(-3,5$ a $-2,9) ; p<0,001$ & 81,0 & 41,5 & $-3,3^{*}(-3,7$ a $-3,0) ; p<0,001$ & 71,5 & 39,5 & $-3,0^{*}(-3,3$ a $-2,8) ; p<0,001$ \\
\hline ES & 98,4 & 46,7 & $-3,6^{*}(-4,3$ a $-2,9) ; p<0,001$ & 108,7 & 51,7 & $-3,5^{*}(-4,0$ a $-3,1) ; p<0,001$ & 94,2 & 45,1 & $-3,5^{*}(-4,2$ a $-2,8) ; p<0,001$ \\
\hline RJ & 101,6 & 42,3 & $-4,5^{*}(-5,3$ a $-3,7) ; p<0,001$ & 113,8 & 47,9 & $-4,3^{*}(-4,9$ a $-3,7) ; p<0,001$ & 97,7 & 40,7 & $-4,5^{*}(-5,8$ a $-3,2) ; p<0,001$ \\
\hline SP & 84,3 & 41,7 & $-3,8^{*}(-4,2$ a $-3,4) ; p<0,001$ & 95,8 & 46,7 & $-3,8^{*}(-4,0$ a $-3,5) ; p<0,001$ & 79,4 & 40,1 & $-3,8^{*}(-4,0$ a $-3,5) ; p<0,001$ \\
\hline Sul & 91,0 & 45,9 & $-3,7^{*}(-4,1$ a $-3,2) ; p<0,001$ & 96,9 & 49,5 & $-3,7^{*}(-4,1$ a $-3,2) ; p<0,001$ & 91,3 & 45,6 & $-3,6^{*}(-4,0$ a $-3,2) ; p<0,001$ \\
\hline PR & 98,9 & 49,9 & $-3,8^{*}(-4,1$ a $-3,6) ; p<0,001$ & 108,0 & 55,3 & $-3,8^{*}(-4,1$ a $-3,5) ; p<0,001$ & 95,5 & 48,0 & $-3,8^{*}(-4,1$ a $-3,5) ; p<0,001$ \\
\hline SC & 89,1 & 37,7 & $-4,4^{*}(-4,8$ a $-4,0) ; p<0,001$ & 94,4 & 39,7 & $-3,9^{*}(-5,9$ a $-2,0) ; p<0,001$ & 89,5 & 38,2 & $-4,3^{*}(-4,7$ a $-3,9) ; p<0,001$ \\
\hline RS & 86,1 & 46,5 & $-3,1^{*}(-4,2 a-1,9) ; p<0,001$ & 88,9 & 49,4 & $-3,0^{*}(-3,6$ a $-2,3) ; p<0,001$ & 89,3 & 47,0 & $-3,4^{*}(-4,1$ a $-2,6) ; p<0,001$ \\
\hline
\end{tabular}

*Significância estatística ( $p<0,05)$. 1 Taxa de mortalidade/100 mil habitantes. AAPC: Average Annual Percent Change; RO: Rondônia; AC: Acre; AM: Amazonas; RR: Roraima; PA: Pará; AP: Amapá; TO: Tocantins; MA: Maranhão; PI: Piauí; CE: Ceará; RN: Rio Grande do Norte; PB: Paraíba; PE: Pernambuco; AL: Alagoas; SE: Sergipe; BA: Bahia; MG: Minas Gerais; ES: Espirito Santo; RJ: Rio de Janeiro; SP: São Paulo; PR: Paraná; SC: Santa Catarina; RS: Rio Grande do Sul; MS: Mato Grosso do Sul; MT: Mato Grosso; GO: Goiás; DF: Distrito Federal.

corroborando outros estudos. ${ }^{4,23}$ As taxas de mortalidades foram maiores nas regiões Sudeste e Sul, porém com tendência de redução significativa. Em contraste, o Norte e o Nordeste apresentaram as menores taxas, mas com tendência de crescimento significativo ao longo da série histórica. Esse contexto epidemiológico-espacial heterogêneo é resultado das diferenças sociais, econômicas, demográficas e epidemiológicas existentes entre as regiões. Em virtude disso, os achados devem ser analisados sob a ótica de três dimensões: i) transição demográfica e epidemiológica; ii) determinantes sociais da saúde; e iii) qualidade dos sistemas de informações. 
Índice de Desenvolvimento Humano (IDH)

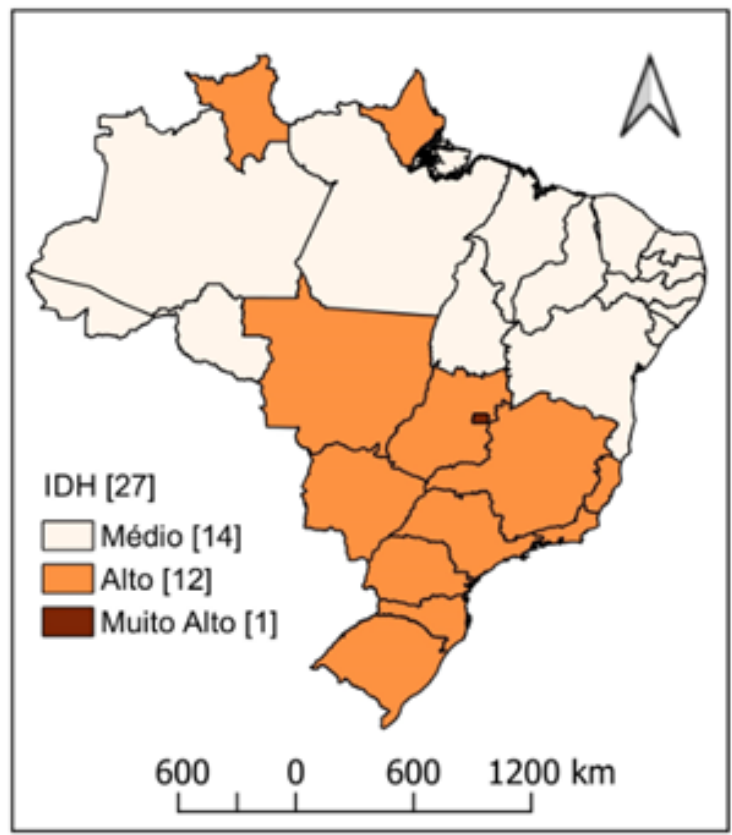

Índice de Vulnerbilidade Social (IVS)

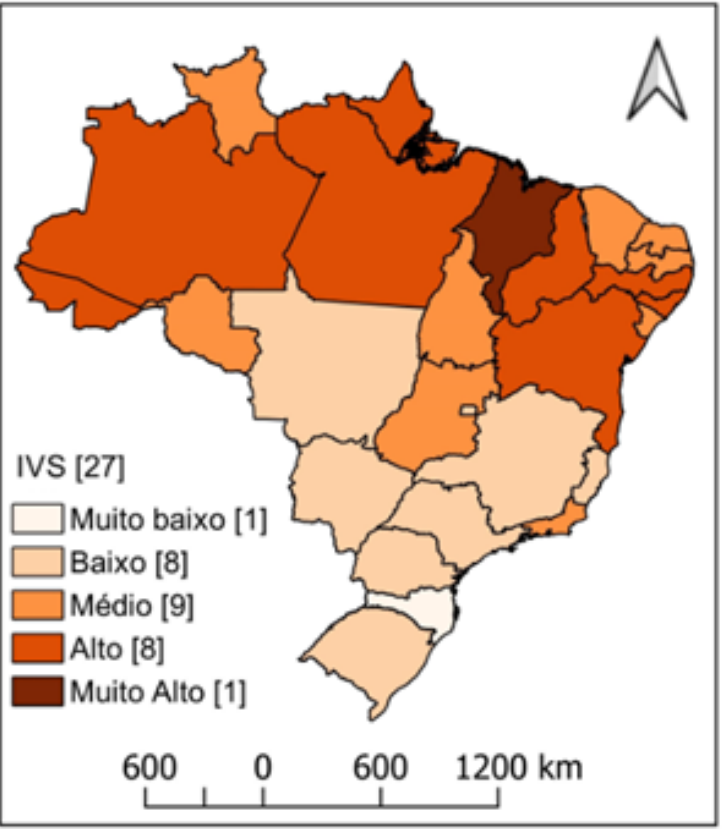

Figura 2 - Distribuição espacial do índice de desenvolvimento humano (IDH) e do índice de vulnerabilidade social (IVS) nos estados brasileiros. Brasil (2010).

Desde os anos 1940, o Brasil tem passado por importantes transformações demográficas: redução da taxa de mortalidade geral e o declínio da natalidade resultaram em grandes mudanças no regime demográfico e na estrutura etária da população, com significativo aumento de idosos. ${ }^{24} \mathrm{Em} 2000$, essa população era de pouco mais de 14,2 milhões, passando para 19,6 milhões em 2010, devendo alcançar 41,5 milhões já em 2030, ${ }^{25}$ com maior concentração das regiões Sudeste e Sul. O impacto do processo de envelhecimento populacional no padrão de morbimortalidade é significativo, uma vez que implica aumento das doenças crônicas, ${ }^{26}$ dentre as quais se destacam as DCBV. Em nosso estudo, 77,8\% dos óbitos ocorreram em idosos.

Estudos apontam que o risco de mortalidade por DCBV na população idosa é substancialmente maior do que em outras faixas etárias. Uma das razões é o acúmulo de fatores de risco, como hipertensão, diabetes, dislipidemias, etilismo, tabagismo e hábitos alimentares inadequados. ${ }^{27,28} \mathrm{Em}$ nível nacional, por exemplo, a prevalência de hipertensão pode acometer $68 \%$ dos idosos. ${ }^{29}$

Ademais, o processo de transição demográfica ocorre em concomitância com uma segunda transição, a epidemiológica, caracterizada pelas mudanças no perfil de adoecimento da população. ${ }^{30}$ Nas últimas décadas, tem sido observado declínio das doenças infecciosas e parasitárias e aumento da ocorrência das crônico-degenerativas, muitas das quais, conforme mencionado anteriormente, elevam o risco de mortalidade por DCBV. ${ }^{27}$ As regiões Norte e Nordeste são as mais expostas à vulnerabilidade social e ao menor desenvolvimento humano, resultando em maior mortalidade por doenças decorrentes desse contexto social desfavorável e menor participação das DCBV, diferentemente do que se observa nas regiões mais desenvolvidas do país (Sudeste e Sul). Nesse sentido, as maiores taxas observadas nos estados mais desenvolvidos refletem as diferenças sociais e, consequentemente, maior participação das condições crônicas no perfil de mortalidade. Em contrapartida, regiões mais vulneráveis podem apresentar menores taxas em razão da persistência da mortalidade em decorrência de doenças relacionadas à pobreza. ${ }^{32}$

As duas transições não ocorrem de modo homogêneo no Brasil, havendo um descompasso entre as regiões. ${ }^{31} \mathrm{~A}$ transição desigual explica, em partes, as diferenças entre as regiões brasileiras no tocante à mortalidade por DCBV. Esse cenário justifica a associação positiva entre a mortalidade por DCBV e o desenvolvimento humano e a associação negativa desta com a vulnerabilidade social, o que representa a influência do contexto epidemiológico e social no perfil de mortalidade da população.

Todavia, a análise isolada das taxas não é suficiente para compreender a dinâmica epidemiológica das DCBV, sendo necessário refletir sobre a sua tendência ao longo da série temporal. Nas regiões Norte e Nordeste, em geral, as taxas mostraram padrão temporal de crescimento e, nas regiões Sudeste e Sul, observou-se declínio. Esses achados refletem a influência dos determinantes sociais da saúde no padrão de mortalidade por DCBV. As condições socioeconômicas, incluindo desenvolvimento humano, condição de renda e situação educacional, exercem influência significativa no risco de um indivíduo morrer por esse grupo de doenças. , $^{4,633-35}$ 
Tabela 3 - Percentual de variação anual das taxas de mortalidade padronizadas por DCBV, segundo gênero - Brasil (1996-2015)

\begin{tabular}{|c|c|c|c|c|c|c|}
\hline \multirow{2}{*}{$\begin{array}{l}\text { Unidade } \\
\text { espacial }\end{array}$} & \multicolumn{2}{|c|}{ Ambos os gêneros } & \multicolumn{2}{|r|}{ Masculino } & \multicolumn{2}{|r|}{ Feminino } \\
\hline & Período & $\begin{array}{c}\text { APC (IC 95\%) } \\
\text { p valor }\end{array}$ & Período & $\begin{array}{c}\text { APC (IC 95\%) } \\
\text { p valor }\end{array}$ & Período & $\begin{array}{l}\text { APC (IC 95\%) } \\
\text { p valor }\end{array}$ \\
\hline Norte & $1996-2015$ & $0,4^{*}(0,0$ a 0,8$) ; p<0,001$ & $1996-2015$ & $0,7^{*}(0,3$ a 1,1$) ; p<0,001$ & $1996-2015$ & $0,1(-0,3$ a 0,5$) ; p=0,6$ \\
\hline RO & $1996-2015$ & $-1,8^{\star}(-2,2 a-1,8) ; p<0,001$ & $1996-2015$ & $1,6^{*}(-2,3 a-1,0) ; p<0,001$ & $1996-2015$ & $-1,9^{*}(-2,3 a-1,4) ; p<0,001$ \\
\hline \multirow{4}{*}{$A C$} & 1996-1999 & $-16,2(-30,7$ a 1,4$) ; p=0,1$ & $1996-2002$ & $-5,6^{*}(-9,4 a-1,7) ; p<0,001$ & 1996-1999 & $-16,2(-30,7$ a 1,4$) ; p=0,1$ \\
\hline & $1999-2006$ & $9,9^{*}(3,1$ a 17,2$) ; p<0,001$ & $2002-2006$ & $13,2^{*}(0,5$ a 27,5$) ; p<0,001$ & $1999-2006$ & $9,9^{*}(3,1$ a 17,2$) ; p<0,001$ \\
\hline & 2006-2011 & $-6,4(-17$ a 5,6$) ; p=0,2$ & \multirow{2}{*}{$2006-2015$} & \multirow{2}{*}{$-0,8(-2,9$ a 1,4$) ; p=0,4$} & 2006-2011 & $-6,4(-17,0$ a 5,6$) ; p=0,2$ \\
\hline & $2011-2015$ & $7,1(-5,0$ a 20,8$) ; p=0,2$ & & & $2011-2015$ & $7,1(-5,0$ a 30,8$) ; p=0,2$ \\
\hline AM & $1996-2015$ & $0,6^{*}(0,2$ a 1,1$) ; p<0,001$ & $1996-2015$ & $0,9^{*}(0,2$ a 1,5$) ; p<0,001$ & $1996-2015$ & $0,4(-0,1$ a 1,0$) ; p=0,1$ \\
\hline RR & $1996-2015$ & $-2,2^{*}(-3,0 a-1,3) ; p<0,001$ & $1996-2015$ & $-2,0^{*}(-3,6 a-0,4) ; p<0,001$ & $1996-2015$ & $-2,3^{*}(-3,3 a-1,3) ; p<0,001$ \\
\hline \multirow{4}{*}{ PA } & $1996-2004$ & $-0,8(-2,9$ a 1,2$) ; p=0,9$ & 1996-1998 & $11,6(-1,1$ a 25,9$) ; p=0,1$ & \multirow{2}{*}{$1996-2004$} & \multirow{2}{*}{$-0,8(-2,9$ a 1,2$) ; p=0,4$} \\
\hline & $2004-2008$ & $6,3(-3,3$ a 16,8$) ; p=1,4$ & $1998-2001$ & $-4,2(-15,1$ a 8,2$) ; p=0,4$ & & \\
\hline & $2008-2015$ & $23(-47$ ? 1 & $2001-2008$ & $4,3^{*}(2,2$ a 6,4$) ; p<0,001$ & $2004-2008$ & $6,3(-3,3$ a 16,8$) ; p=0,2$ \\
\hline & $2000-\angle 010$ & 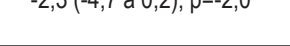 & $2008-2015$ & $-0,5(-2,1$ a 1,2$) ; p=0,5$ & $2008-2015$ & $-2,3(-4,7$ a 0,2$) ; p=0,1$ \\
\hline \multirow{3}{*}{ AP } & $1996-2007$ & $-5,8^{*}(-8,8$ a $-2,7) ; p=0,6$ & $1996-2002$ & $1,9(-4,3$ a 8,4$) ; p=0,5$ & $1996-2007$ & $-5,8^{*}(-8,8$ a $-2,7) ; p<0,001$ \\
\hline & \multirow{2}{*}{$2007-2015$} & \multirow{2}{*}{$5,5^{*}(0,1$ a 11,3$) ; p<0,001$} & $2002-2006$ & $-11,1(-26,1$ a 7,0$) ; p=0,2$ & \multirow{2}{*}{$2007-2015$} & \multirow{2}{*}{$5,5^{\star}(0,1$ a 11,3$) ; p<0,001$} \\
\hline & & & $2006-2015$ & $2,4(-1,0$ a 5,9$) ; p=0,2$ & & \\
\hline \multirow{3}{*}{ TO } & $1996-2003$ & $11,6^{*}(7,5$ a 15,8$) ; p<0,001$ & $1996-2000$ & $3,1(-2,5$ a 8,9$) ; p=0,3$ & $1996-2003$ & $11,6^{*}(7,5$ a 15,8$) ; p<0,001$ \\
\hline & \multirow{2}{*}{$2003-2015$} & \multirow{2}{*}{$-2,8^{*}(-4,4$ a $-1,1) ; p<0,001$} & $2000-2003$ & $15,3(-3,1$ a 37,3$) ; p=0,1$ & \multirow{2}{*}{$2003-2015$} & \multirow{2}{*}{$-2,8^{*}(-4,4 a-1,1) ; p<0,001$} \\
\hline & & & 2003-2015 & $-2,3^{*}(-3,3 a-1,3) ; p<0,001$ & & \\
\hline \multirow{4}{*}{ Nordeste } & $1996-2003$ & $1,7^{*}(0,5$ a 2,9$) ; p=0,3$ & $1996-2003$ & $2,1^{*}(-0,9$ a 3,3$) ; p=0,1$ & $1996-2003$ & $1,4^{*}(0,1$ a 2,7$) ; p<0,001$ \\
\hline & $2003-2006$ & $7,4(-1,5$ a 17,2$) ; p=1,9$ & $2003-2006$ & $6,6(-2,3$ a 16,4$) ; p=0,1$ & $2003-2006$ & $8,3(-1,5$ a 19,1$) ; p=0,1$ \\
\hline & $2006-2010$ & $-4,5^{*}(-8,5$ a $-0,2) ; p=0,3$ & $2006-2010$ & $-3,7(-7,8$ a 0,6$) ; p=0,1$ & $2006-2010$ & $-5,1^{*}(-9,5 a-0,5) ; p<0,001$ \\
\hline & $2010-2015$ & $0,3(-1,7$ a 2,2$) ; p=0,3$ & $2010-2015$ & $1,1(-0,9$ a 3,1$) ; p=0,2$ & $2010-2015$ & $-0,4(-2,5$ a 1,8$) ; p=0,7$ \\
\hline \multirow{3}{*}{ MA } & $1996-2006$ & $4,5^{*}(2,2$ a 6,9$) ; p<0,001$ & $1996-2000$ & $0,1(-7,1$ a 7,8$) ; p=1,0$ & $1996-2003$ & $4,5^{*}(2,2$ a 6,9$) ; p<0,001$ \\
\hline & $2003-2006$ & $18,3(-0,2$ a 40,2$) ; p=0,1$ & $2000-2007$ & $13,4^{*}(8,9$ a 18,0$) ; p<0,001$ & $2003-2006$ & $18,3(-0,2$ a 40,2$) ; p=0,1$ \\
\hline & $2006-2015$ & $-0,2(-1,7$ a 1,4$) ; p=0,8$ & $2007-2015$ & $-0,1(-2,6$ a 2,6$) ; p=1,0$ & $2006-2015$ & $-0,2(-1,7$ a 1,4$) ; p=0,8$ \\
\hline \multirow{2}{*}{ PI } & $1996-2006$ & $8,9^{*}(7,6$ a 10,3$) ; p<0,001$ & $1996-2007$ & $8,4^{*}(6,8$ a 10,1$) ; p<0,001$ & $1996-2006$ & $8,9^{*}(7,6$ a 10,3$) ; p<0,001$ \\
\hline & $2006-2015$ & $-1,3(-2,7$ a 0,2$) ; p=0,1$ & $2007-2015$ & $-1,4(-3,7$ a 1,1$) ; p=0,2$ & $2006-2015$ & $-1,3(-2,7$ a 0,2$) ; p=0,1$ \\
\hline & $1996-2007$ & $2,8^{*}(1,7$ a 3,9$) ; p<0,001$ & 1996-1998 & $11,5(-2,2$ a 27,0$) ; p=0,1$ & $1996-2007$ & $2,8^{*}(1,7$ a 3,9$) ; p<0,001$ \\
\hline CE & 20072015 & $20 *(30001) \cdot 0=0001$ & $1998-2008$ & $2,0^{*}(0,8$ a 3,2$) ; p<0,001$ & 20072015 & $n=0001$ \\
\hline & $2001-2015$ & $-2,2(-3,9 a-0,4) ; p<0,001$ & $2008-2015$ & $-1,2(-2,9$ a 0,5$) ; p=0,1$ & $2001-2015$ & $\begin{array}{lll}<0,001 \\
\end{array}$ \\
\hline RN & $1996-2009$ & $2,4^{*}(1,4$ a 3,5$) ; p<0,001$ & $1996-2008$ & $4,2^{*}(2,9$ a 5,4$) ; p<0,001$ & $1996-2009$ & $2,4^{*}(1,4$ a 3,5$) ; p<0,001$ \\
\hline RIV & $2009-2015$ & $-3,9^{*}(-7,0$ a $-0,6) ; p<0,001$ & $2008-2015$ & $-3,3^{*}(-5,9$ a $-0,6) ; p<0,001$ & $2009-2015$ & $-3,9^{*}(-7,0$ a $-0,6) ; p<0,001$ \\
\hline & 1996-1998 & $-11,8^{*}(-22,1$ a 0,0$) ; p<0,001$ & 1996-1999 & $-5,5(-12,3$ a 2,3$) ; p=0,1$ & 1996-1998 & $-11,8^{*}(-22,1$ a 0,0$) ; p<0,001$ \\
\hline PB & $1998-2007$ & $8,9^{*}(7,4$ a 10,4$) ; p<0,001$ & 1999-2007 & $10,6^{*}(8,3 \mathrm{~A} 13,0) ; p<0,001$ & $1998-2007$ & $8,9^{*}(7,4$ a 10,4$) ; p<0,001$ \\
\hline & $2007-2015$ & $-3,1^{*}(-4,4$ a $-1,8) ; p<0,001$ & $2007-2015$ & $-3,5^{*}(-5,1$ a $-1,8) ; p<0,001$ & $2007-2015$ & $-3,1^{*}(-4,4 a-1,8) ; p<0,001$ \\
\hline & 1996-1998 & $5,1(-2,8$ a 13,7$) ; p=0,2$ & $1996-2006$ & $1,1^{*}(0,3$ a 2,0$) ; p<0,001$ & 1996-1998 & $5,1(-2,8$ a 13,7$) ; p=0,2$ \\
\hline$P E$ & $1998-2001$ & $-6,0(-13,1$ a 1,7$) ; p=0,1$ & & & $1998-2001$ & $-6,0(-13,1$ a 1,7$) ; p=0,1$ \\
\hline PE & $2001-2005$ & $4,9^{*}(0,9$ a 9,1$) ; p<0,001$ & 2006-2015 & $-2,0^{*}(-3,0 a-1,1) ; p<0,001$ & $2001-2005$ & $4,9^{*}(0,9$ a 9,1$) ; p<0,001$ \\
\hline & $2005-2015$ & $-3,2^{*}(-3,8$ a $-2,6) ; p<0,001$ & & & $2005-2015$ & $-3,2^{*}(-3,8$ a $-2,6) ; p<0,001$ \\
\hline at & $1996-2007$ & $2,4^{*}(1,2$ a 3,7$) ; p<0,001$ & $1996-2007$ & $3,2^{*}(2,4$ a 4,0$) ; p<0,001$ & $1996-2007$ & $2,4^{*}(1,2$ a 3,7$) ; p<0,001$ \\
\hline AL & $2007-2015$ & $-2,1(-4,0$ a $-0,1) ; p<0,001$ & $2007-2015$ & $-1,5^{*}(-2,7 a-0,3) ; p<0,001$ & $2007-2015$ & $-2,1^{*}(-4,0$ a $-0,1) ; p<0,001$ \\
\hline$c \Gamma^{-}$ & $1996-2005$ & $5,7^{*}(4,0$ a 7,5$) ; p<0,001$ & $1996-2005$ & $5,8^{*}(4,1$ a 7,4$) ; p<0,001$ & $1996-2005$ & $5,7^{*}(4,0$ a 7,5$) ; p<0,001$ \\
\hline SE & $2005-2015$ & $-2,1^{*}(-3,5 a-0,7) ; p<0,001$ & 2005-2015 & $-1,4^{*}(-2,7$ a $-0,1) ; p<0,001$ & $2005-2015$ & $-2,1^{*}(-3,5$ a $-0,7) ; p<0,001$ \\
\hline BA & $1996-2015$ & $-0,0(-0,6$ a 0,5$) ; p=0,9$ & $1996-2015$ & $0,2(-0,3$ a 0,8$) ; p=0,4$ & $1996-2015$ & $-0,2(-0,8$ a 0,3$) ; p=0,4$ \\
\hline
\end{tabular}




\begin{tabular}{|c|c|c|c|c|c|c|}
\hline \multicolumn{7}{|c|}{ Continuação da tabela } \\
\hline \multirow{3}{*}{$\begin{array}{l}\text { Centro- } \\
\text { Oeste }\end{array}$} & \multirow{3}{*}{$1996-2015$} & \multirow{3}{*}{$-2,8^{*}(-3,4$ a $-2,2) ; p<0,001$} & \multirow{3}{*}{$1996-2015$} & \multirow{3}{*}{$-2,7^{*}(-3,3$ a $-2,2) ; p<0,001$} & $1996-2005$ & $-0,5(-2,0$ a 1,0$) ; p=0,5$ \\
\hline & & & & & $2005-2008$ & $-0,9(-23,1$ a 7,7$) ; p=0,2$ \\
\hline & & & & & $2008-2015$ & $-1,6(-3,8$ a 0,6$) ; p=0,1$ \\
\hline MS & $1996-2015$ & $-2,4^{*}(-2,9$ a $-2,0) ; p<0,001$ & $1996-2015$ & $-2,4^{*}(-3,0 a-1,8) ; p<0,001$ & $1996-2015$ & $-2,4^{*}(-2,8$ a 1,9$) ; p<0,001$ \\
\hline \multirow{3}{*}{ MT } & \multirow{3}{*}{$1996-2015$} & \multirow{3}{*}{$-1,9^{*}(-3,0$ a $-0,8) ; p<0,001$} & $1996-1998$ & $9,7(-3,5$ a 24,6$) ; p=0,1$ & \multirow{3}{*}{$1996-2015$} & \multirow{3}{*}{$-2,5^{*}(-3,2 a-1,9) ; p<0,001$} \\
\hline & & & $1998-2010$ & $-2,3^{*}(-3,1 a-1,4) ; p<0,001$ & & \\
\hline & & & $2010-2015$ & $-5,9^{*}(-8,6$ a $-3,2) ; p<0,001$ & & \\
\hline \multirow{3}{*}{ GO } & \multirow{3}{*}{$1996-2015$} & \multirow{3}{*}{$-2,2^{*}(-2,6$ a $-1,8) ; p<0,001$} & 1996-1999 & $2,9(-3,0$ a 9,1$) ; p<0,001$ & \multirow{3}{*}{$1996-2015$} & \multirow{3}{*}{$-2,2(-2,6$ a $-1,8) ; p<0,001$} \\
\hline & & & $1999-2007$ & $-3,8^{*}(-5,3$ a $-2,3) ; p<0,001$ & & \\
\hline & & & $2007-2015$ & $-0,9(-2,1$ a 0,4$) ; p=0,2$ & & \\
\hline \multirow{2}{*}{ DF } & 1996-1998 & $5,7(-14,4$ a 30,6$) ; p=0,6$ & \multirow{2}{*}{$1996-2015$} & \multirow{2}{*}{$-4,0^{*}(-4,6$ a $-3,4) ; p<0,001$} & 1996-1998 & $5,7(-14,4$ a 30,6$) ; p=0,6$ \\
\hline & $1998-2015$ & $-4,4^{*}(-5,1$ a $-3,7) ; p<0,001$ & & & $1998-2015$ & $-4,4^{*}(-5,1$ a $-3,7) ; p<0,001$ \\
\hline Sudeste & $1996-2015$ & $-3,8^{*}(-4,1$ a $-3,4) ; p<0,001$ & $1996-2015$ & $-3,8^{*}(-4,2$ a $-3,5) ; p<0,001$ & $1996-2015$ & $-3,8^{*}(-4,2$ a $-3,4) ; p<0,001$ \\
\hline \multirow{2}{*}{ MG } & 1996-2009 & $-2.6^{*}(-3,2 a-1,9) ; p<0,001$ & \multirow{2}{*}{$1996-2015$} & \multirow{2}{*}{$-3,3^{*}(-3,7$ a $-3,0) ; p<0,001$} & \multirow{2}{*}{$1996-2015$} & \multirow{2}{*}{$-3,0^{*}(-3,3$ a $-2,8) ; p<0,001$} \\
\hline & $2009-2015$ & $-5,5^{*}(-7,4$ a $-3,5) ; p<0,001$ & & & & \\
\hline \multirow{2}{*}{ ES } & \multirow{2}{*}{$1996-2015$} & \multirow{2}{*}{$-3,6(-4,3$ a $-2,9) ; p<0,001$} & \multirow{2}{*}{$1996-2015$} & \multirow{2}{*}{$-3,5^{*}(-4,0$ a $-3,1) ; p<0,001$} & $1996-2009$ & $-2,6^{*}(-3,2 a-1,6) ; p<0,001$ \\
\hline & & & & & $2009-2015$ & $-5,5^{*}(-7,4$ a $-3,5) ; p<0,001$ \\
\hline \multirow{3}{*}{ RJ } & 1996-2005 & $-5,1^{*}(-5,9 a-4,4) ; p<0,001$ & 1996-2010 & $-3,9^{*}(-4,4$ a $-3,5) ; p<0,001$ & $1996-2005$ & $-5,1^{*}(-5,9$ a $-4,4) ; p<0,001$ \\
\hline & $2005-2008$ & $-0,6(-8,9$ a 8,4$) ; p=0,9$ & 20102015 & $51 *\left(710^{2} 202\right) \cdot n=0001$ & $2005-2008$ & $-0,6^{*}(-8,9$ a 8,4$) ; p<0,001$ \\
\hline & $2008-2015$ & $-5,4^{*}(-6,5 a-4,3) ; p<0,001$ & $2010-2010$ & $-0,4 \quad(-1,4 \quad a-0,0), p=0,001$ & $2008-2015$ & $-5,4^{*}(-6,5 a-4,3) ; p<0,001$ \\
\hline SP & $1996-2015$ & $-3,8^{*}(-4,2$ a $-3,4) ; p<0,001$ & $1996-2015$ & $-3,8^{*}(-4,0$ a $-3,5) ; p<0,001$ & $1996-2015$ & $-3,8^{*}(-4,0$ a $-3,5) ; p<0,001$ \\
\hline Sul & $1996-2015$ & $-3,7^{\star}(-4,1$ a $-3,2) ; p<0,001$ & 1996-2015 & $-3,7^{*}(-4,1$ a $-3,2) ; p<0,001$ & $1996-2015$ & $-3,6^{*}(-4,0$ a $-3,2) ; p<0,001$ \\
\hline PR & $1996-2015$ & $-3,8^{*}(-4,1$ a $-3,6) ; p<0,001$ & $1996-2015$ & $-3,8^{*}(-4,1$ a $-3,5) ; p<0,001$ & $1996-2015$ & $-3,8^{*}(-4,1$ a $-3,5) ; p<0,001$ \\
\hline & & & $1996-1998$ & $5,2(-9,2$ a 21,9$) ; p=0,5$ & & \\
\hline SC & $1996-2015$ & $-4,4^{*}(-4,8$ a $-4,0) ; p<0,001$ & $1998-2002$ & $-8,3^{*}(-14,8$ a $-1,3) ; p<0,001$ & $1996-2015$ & $-4,3^{*}(-4,7$ a $-3,9) ; p<0,001$ \\
\hline & & & $2002-2015$ & $-3,9^{*}(-4,7$ a $-3,2) ; p<0,001$ & & \\
\hline & $1996-2012$ & $-3,0^{*}(-3,0$ a $-3,4) ; p<0,001$ & 1996-1998 & $4,7(-0,0$ a 9,6$) ; p<0,001$ & & \\
\hline RS & & & $1998-2006$ & $-3,9^{*}(-4,5$ a $-3,3) ; p<0,001$ & 1996-2012 & $-3,0^{*}(-3,4$ a $-2,6) ; p<0,001$ \\
\hline Ro & $2012-2015$ & $-5,4^{*}(-10,0 a-0,6) ; p<0,001$ & $2006-2010$ & $-1,6(-3,8$ a 0,7$) ; p=0,1$ & & \\
\hline & & & $2010-2015$ & $-5,5^{*}(-6,4$ a $-4,5) ; p<0,001$ & 15 & 01 \\
\hline
\end{tabular}

*Significância estatistica (p<0,05). APC: Annual Percent Change; RO: Rondônia; AC: Acre;AM: Amazonas; RR: Roraima; PA: Pará;AP: Amapá; TO: Tocantins; MA: Maranhão; Pl: Piauí; CE: Ceará; RN: Rio Grande do Norte; PB: Paraiba; PE: Pernambuco; AL: Alagoas; SE: Sergipe; BA: Bahia; MG: Minas Gerais; ES: Espírito Santo; RJ: Rio de Janeiro; SP: São Paulo; PR: Paraná; SC: Santa Catarina; RS: Rio Grande do Sul; MS: Mato Grosso do Sul; MT: Mato Grosso; GO: Goiás; e DF: Distrito Federal.

Tabela 4 - Modelo de regressão (ordinay least square [OLS]) entre a taxa de mortalidade por DCBV e o índice de desenvolvimento humano e índice de vulnerabilidade social - Brasil (1996-2015)

\begin{tabular}{lccc}
\hline Variável & Coeficiente & Estatística t & p valor \\
\hline Índice de desenvolvimento humano municipal (IDHM) & 61,588 & 2,091 & $0,046^{*}$ \\
\hline IDHM longevidade & 90,265 & 1,866 & 0,073 \\
\hline IDHM educação & 47,075 & 1,861 & 0,074 \\
\hline IDHM renda & 56,476 & 2,301 & $0,029^{*}$ \\
\hline Índice de vulnerabilidade social (IVS) & $-40,802$ & $-2,353$ & $0,026^{*}$ \\
\hline IVS infraestrutura urbana & $-15,998$ & $-1,110$ & 0,277 \\
\hline IVS capital humano & $-31,883$ & $-2,092$ & $0,046^{*}$ \\
\hline IVS renda e trabalho & $-35,322$ & $-2,528$ & $0,018^{*}$ \\
\hline
\end{tabular}

*Associação significativa. 
Recente estudo da Carga Global de Doenças (Global Burden of Disease, 2015) mostrou que os estados brasileiros situados no tercil mais baixo do índice de desenvolvimento social apresentaram reduções menores das taxas de mortalidade, quando comparados aos estados situados no tercil superior de desenvolvimento. ${ }^{4}$ Nesse mesmo estudo, o tercil inferior foi composto, unicamente, por estados das regiões Norte e Nordeste. Sugere-se, portanto, que as melhores condições de vida apresentam dupla influência na tendência da mortalidade: i) reduzem os fatores de risco para a ocorrência dos eventos de doença; e ii) contribuem para a sobrevida dos pacientes quando tais eventos ocorrem, reduzindo a chance de ocorrência do óbito.

Por fim, é necessário refletir ainda sobre a qualidade dos registros de mortalidade, sendo este um desafio para o adequado monitoramento das condições de saúde da população. O inadequado preenchimento da declaração de óbito, resultando em elevado número de códigos garbage, as dificuldades em realizar investigação epidemiológica dos óbitos registrados com causa mal definida e a falta de recursos humanos capacitados para atuação nos serviços de vigilância do óbito são problemas comuns evidenciados em todo o país, embora as regiões Norte e Nordeste sejam as mais atingidas pelo problema. ${ }^{36,37} \mathrm{~A}$ qualidade duvidosa das informações configura-se como uma importante limitação deste estudo.

Entre 1996 e 2005, o percentual de óbitos com causas mal definidas nessas regiões foi superior a $20 \%$, sendo ainda maior na população idosa quando comparada com outras faixas etárias. ${ }^{38}$ Nesse sentido, as taxas de mortalidade no Norte e Nordeste, por exemplo, podem ser superiores àquelas que evidenciamos neste estudo. Por outro lado, é necessário destacar que, nos últimos anos, importantes avanços na qualidade da informação foram observados nessas regiões. ${ }^{13}$

\section{Referências}

1. World Health Organization. (WHO). Health statistics and information systems. Estimates for 2000-2016. [Internet]. Genebra: WHO; 2018. [Acesso em 21 out 2018].Disponível em: http://www.who.int/healthinfo/ global_burden_disease/estimates/en/index1.html.

2. Malta DC, França E, Abreu DMX, Perillo RD, Salmen MC, Teixeira RA, et al. Mortality due to noncommunicable diseases in Brazil, 1990 to 2015, according to estimates from the Global Burden of Disease study. São Paulo Med J. 2017;135(3):213-21.

3. World Health Organization. The top 10 causes of death [Internet]. Genebra: WHO; 2018. [Acesso em 22 oute 2018]. Disponível em: http://www.who. int/news-room/fact-sheets/detail/the-top-10-causes-of-death.

4. Lotufo PA, Goulart AC, Passos VMA, Satake FM, Souza MFM, França EB, et al. Cerebrovascular disease in Brazil from 1990 to 2015: Global Burden of Disease 2015. Rev Bras Epidemiol. 2017;20(1):129-41.

5. Alwan A, Maclean DR, Riley LM, d'Espaignet ET, Mathers CD, Stevens $\mathrm{GA}$, et al. Monitoring and surveillance of chronic non-communicable diseases: progress and capacity in high-burden countries. Lancet. 2010;376(9755):1861-8.

6. Vincens N, Stafström M. Income inequality, economic growth and stroke mortality in Brazil: longitudinal and regional analysis 2002-2009. PLoS One. 2015;10(9):e0137332.

\section{Conclusão}

Conclui-se que a mortalidade por DCBV no Brasil apresenta comportamento epidemiológico desigual entre as regiões. As maiores taxas foram observadas nos estados com melhor grau de desenvolvimento humano e menor vulnerabilidade social, mas com tendência decrescente ao longo da série temporal. Por outro lado, nos estados com menor desenvolvimento e maior vulnerabilidade, as taxas foram menores, mas com tendência de crescimento. Nesse sentido, advogamos que as políticas públicas devem ser desenvolvidas considerando o contexto regional/local.

\section{Contribuição dos autores}

Concepção e desenho da pesquisa estudo: Souza CDF, Santos CD, Pereira MC, Paiva JPS, Leal TC, Silva LF, Araújo AKBF; Obtenção dos dados: Souza CDF, Silva LF, Mariano RS, Paiva JPS; Análise e interpretação dos dados, Redação do manuscrito e Revisão crítica do manuscrito quanto ao conteúdo intelectual importante: Souza CDF, Santos CD, Pereira MC, Paiva JPS, Leal TC, Silva LF, Araújo AKBF, Baggio JAO, Oliveira DJ, Mariano RS; Análise estatística: Souza CDF, Silva LF.

\section{Potencial conflito de interesses}

Declaro não haver conflito de interesses pertinentes.

\section{Fontes de financiamento}

O presente estudo não teve fontes de financiamento externas.

\section{Vinculação acadêmica}

Não há vinculação deste estudo a programas de pósgraduação.

7. Brasil. Ministério da Saúde. Sistema de informações sobre mortalidade (SIM). [Acesso em 13 de agosto 2018]. Disponível em: http://datasus.saude.gov.br/.

8. Organização Mundial da Saúde. Classificação estatística internacional de doenças e problemas relacionados à saúde- CID 10. $10^{\text {a }}$ revisão. São Paulo: Centro Colaborador da Organização Mundial da saúde para a Classificação de Doenças em Português. Brasília, DF: OMS; 1995.

9. Instituto Brasileiro de Geografia e Estatística. Sistema de Recuperação automática de dados-SIDRA. [Acesso em 15 de julho 2018]. Disponível em: https://sidra.ibge.gov.br/home/ipp/Brasil.

10. Kim HJ, Fay MP, Feuer EJ, Midthune DN. Permutation tests for joinpoint regression with applications to cancer rates. Stat Med. 2000;19(3):335-51.

11. Lavados PM, Hennis AJ, Fernandes JG, Medina MT, Legetic B, Hoppe A, et al. Stroke epidemiology, prevention, and management strategies at a regional level: Latin America and the Caribbean. Lancet Neurol. 2007;6(4):362-72.

12. Soares GP, Brum JD, Oliveira GM, Klein CH, Silva NAS. Mortalidade por todas as causas e por doenças cardiovasculares em três estados do Brasil, 1980 a 2006. Rev Panam Salud Publica. 2010;28(4):258-66.

13. Garritano CR, Luz PM, Pires MLE, Barbosa MTS, Batista KM. Analysis of the mortality trend due to cerebrovascular accident in Brazil in the XXI century. Arq Bras Cardiol. 2012;98(6):519-27. 
14. Feigin $\mathrm{VL}$, Abajobir AA, Abate KH, Adc-Allah F, Abdulle AM, Abera SF, et al. Global, regional, and national burden of neurological disorders during 19902015: a systematic analysis for the Global Burden of Disease Study 2015. Lancet Neurol. 2017;16(11):877-97.

15. Cabral NL, Gonçalves ARR, Longo AL, Moro CHC, Costa G, Amaral CH, etal. Trends in stroke incidence, mortality and case fatality rates in Joinville, Brazil: 1995-2006. J Neurol Neurosurg Psychiatry. 2009;80:749-54.

16. Malta DC, Morais Neto OL, Silva Junior JB. Presentation of the strategic action plan for coping with chronic diseases in Brazil from 2011 to 2022. Epidemiol Serv Saúde. 2011;20(4):425-38

17. Pinto LF, Giovanella L. The Family Health Strategy: expanding access and reducing hospitalizations due to ambulatory care sensitive conditions (ACSC). Cienc Saúde Coletiva. 2018;23(6):1903-13.

18. Malta DC, Santos MAS, Stopa SR, Vieira JEB, Melo EA, Reis AAC. Family Health Strategy Coverage in Brazil,according to the National Health Survey, 2013. Cienc Saude Coletiva. 2016;21(2):327-38.

19. Rasella D, Harhay MO, Pamponet ML, Aquino R, Barreto ML. Impact of primary health care on mortality from heart and cerebrovascular diseases in Brazil: a nationwide analysis of longitudinal data. BMJ. 2014;349:g4014.

20. Brasil. Ministério da Saúde do Brasil.. Portaria no 665, de 12 de abril de 2012. [Acesso em 16 de set 2018]. Disponível em: http://bvsms.saude.gov.br/bvs/ saudelegis/gm/2012/PRT0665_12_04_2012.html.

21. Brasil. Ministério da Saúde do Brasil. Acidente vascular cerebral. [Acesso em 19 de set 2018]. Disponível em: http://portalms.saude.gov.br/saude-de-a-z/ acidente-vascular-cerebral-avc.

22. Brasil. Ministério da Saúde do Brasil (BR). Plano de Ações Estratégicas para o Enfrentamento das Doenças Crônicas Não Transmissíveis (DCNT) no Brasil 2011-2022. Brasília: Ministério da Saúde; 2011.

23. Guimarães RM, Andrade SSCA, Machado EL, Bahia CA, Oliveira MM, Jacques FVL. Regional differences in cardiovascular mortality transition in Brazil, 1980 to 2012. Diferenças regionais na transição da mortalidade por doenças cardiovasculares no Brasil, 1980 a 2012. Rev Panam Salud Publica. 2015;37(2):83-89.

24. Alves JED. A transição demográfica e a janela de oportunidade. São Paulo: Instituto Fernand Braudel de Economia Mundial; 2008.

25. Instituto Brasileiro de Geografia e estatística. Estudos e análises - Informação Demográfica e Socioeconômica. № 3: Mudança Demográfica no Brasil no Início do Século XXI Subsídios para as projeções da população. Rio de Janeiro: IBGE; 2015.
26. Mendes ACG, Sá DA, Miranda GMD, Lyra TM, Tavares RAW.. The public healthcare system in the context of Brazil's demographic transition: current and future demands. Cad Saúde Pública. 2012;28(5):955-64.

27. Pires SL, Gagliardi RJ, Gorzoni ML. Study of the main risk factors frequencies for ischemic cerebrovascular disease in elderly patients. Arq Neuropsiquiatr. 2004;62(3-B):844-51.

28. Duncan BB, Chor D, Aquino EML, Bensenor IM, Mill JG, Scdmidt MI, et al. Chronic Non-Communicable Diseases in Brazil: priorities for disease management and research.. Rev Saúde Pública. 2012;46(Suppl.1):126-34.

29. Picon RV, Fuchs FD, Moreira LB, Fuchs SC. Prevalence of hypertension among elderly persons in urban Brazil: a systematic review with metaanalysis. Am J Hypertens. 2013;26(4):541-8.

30. Vasconcelos AMN, Gomes MMF. Transição demográfica: a experiência brasileira. Epidemiol Serv Saúde. 2012;21(4):539-48.

31. Schramm JMA, Oliveira AF, Leite IC, Valente JG, Gadelha AMJ, Portela MC, et al. Demographic transition: the Brazilian experience. Cienc Saude Coletiva. 2004;9(4):897-908

32. Araújo JD. Epidemiological Polarization in Brazil. Epidemiol Serv Saúde. 2012;21(4):533-8.

33. Cabral NL, Longo A, Moro C, Ferst P, Oliveira FA, Vieira CV, et al.et al Education level explains differences in stroke incidence among city districts in Joinville, Brazil: a three-year population-based study. Neuroepidemiology. 2011;36(4):258-64.

34. Bensenor IM, Goulart AC, Szwarcwald CL, Vieira MLFP, Malta DC, Lotufo PA. Prevalence of stroke and associated disability in Brazil: National Health Survey-2013. Arq Neuro-Psiquiatr. 2015;73(9):746-50.

35. Lucena DMM, Figueiredo FWS, Sousa LVA, Paiva LS, Almeida TCC, Galego $\mathrm{S}$, et al. Correlation between municipal human development index and stroke mortality: a study of Brazilian capitals. BMC Res Notes. 2018;11:540.

36. Brasil. Ministério da Saúde. Manual para investigação do óbito com causa mal definida. Brasília: Ministério da Saúde; 2009.

37. Ishitanil LH, Teixeira RA, Abreu DMX, Paixão LMMM, França EB. Quality of mortality statistics' information: garbage codes as causes of death in Belo Horizonte, 2011-2013. Rev Bras Epidemiol. 2017;20(1):34-45.

38. Jorge MHPM, Laurenti R, Lima-Costa MF, Gotlieb SLD, Chiavegatto Filho ADP. Brazilian mortality of elderly persons: the question about ill-defined underlying causes of death. Epidemiol Serv Saúde. 2008;17(4):271-81. 